\title{
INTERPOLATION SETS FOR CONVOLUTION MEASURE ALGEBRAS
}

\author{
COLIN C. GRAHAM ${ }^{1}$
}

\begin{abstract}
These are proved: (1) The union of two interpolation sets for a regular commutative convolution measure algebra is not necessarily an interpolation set. (2) There exists a regular commutative convolution measure algebra for which interpolation sets are not necessarily of spectral synthesis, while every singleton is a Ditkin set. (3) For every nondiscrete LCA group $G$, there exist compact interpolation sets for $M(G)$ whose union is not an interpolation set. A tensor algebra method is used.
\end{abstract}

0 . Introduction. An interpolation set (Helson set) for a commutative Banach algebra $\mathscr{A}$ is a closed subset $E$ of the maximal ideal space $X$ of $\mathscr{A}$ such that every continuous function on $E$, vanishing at infinity, is the restriction to $E$ of a Gelfand transform $\hat{f}$ of some $f \in \mathscr{A}$. Körner [7] has given examples of commutative Banach algebras $\mathscr{A}$ for which (i) the union of two interpolation sets is not an interpolation set, and (ii) interpolation sets $E$ do not obey spectral synthesis, that is, for some $f \in \mathscr{A}$ with $f$ zero on $E$, there do not exist $f_{n} \in \mathscr{A}$ such that $\lim \left\|f_{n}-f\right\|_{\mathscr{S}}=0$ and $\hat{f}_{n}=0$ in a neighborhood of $E$. After a preliminary version of this was prepared, we learned that Körner [18] has also constructed a Helson set for $L^{1}(Z)$ which is not a set of spectral synthesis.

This note may be regarded as an extension of Körner's results (in [7], [8]) to convolution measure algebras. In view of the diversity of convolution measure algebras, the first two results are not surprising, since they hold for general Banach algebras. The third result is perhaps more interesting, in view of the Varopoulos-Drury Theorem which says the union of two interpolation sets for $L^{1}(G)$ is an interpolation set ([2], [4], [14]).

This note is organized as follows: notation is in the remainder of this section; the general method is outlined in $\$ 1$. Results (1)-(3) are proved in $\S \S 2-4$ respectively.

We use the notation of Rudin [10]. The Gelfand and Fourier (-Stieltjes) transform of $f$ will be denoted (ambiguously) by $\hat{f}$; which is meant will be clear from context.

Presented to the Society, April 1, 1972; received by the editors May 24, 1972.

AMS (MOS) subject classifications (1970). Primary 43A10, 43A20.

Key words and phrases. Convolution measure algebras, interpolation set, Helson set.

${ }^{1}$ Partially supported by a National Science Foundation grant.

(c) American Mathematical Society 1973 
1. The general method. We suppose that we have two commutative Banach algebras $B_{1}$ and $B_{2}$ with maximal ideal spaces $X_{1}$ and $X_{2}$. The algebra $\mathscr{A}$ will be the closure $B_{1} \hat{\otimes} B_{2}$, of $B_{1} \otimes B_{2}$ in the greatest cross (projective) norm.

It is clear (cf. [15, p. 58]) that $X=X_{1} \times X_{2}$ is the maximal ideal space of $\mathscr{A}$ and that $\mathscr{A}^{\wedge}$ is regular on $X$ if $B_{1}$ and $B_{2}$ are both regular. If $K_{1}$, $K_{2} \subseteq X_{1}$ are compact interpolation sets for $B_{1}$ and $\varphi_{j}: K_{j} \rightarrow X_{2}$ is a homeomorphism such that there exist $f_{1}, f_{2} \in B_{2}$ for which $\hat{f}_{j} \equiv 1$ on $\varphi_{j}\left(K_{j}\right), j=1$, 2 , then $E_{j}=\left\{\left(k, \varphi_{j}(k): k \in K_{j}\right\}, j=1,2, \cdots\right.$, will be interpolation sets for $\mathscr{A}$ because of the behavior in the first coordinate. The second coordinate will provide the necessary "bad" behavior.

Before proceeding, we need some facts about $L^{1}(0,1)$, which is given the convolution multiplication induced by $x \cdot y=\max (x, y) .\left(L^{1}(0,1)\right.$ is also known as the absolutely continuous functions of bounded variation.) The first is well known [5].

Proposition 1.1. $L^{1}(0,1)$ is a commutative Banach algebra with maximal ideal space $(0,1], \hat{f}(x)=\int_{0}^{x} f(t) d t$ gives the Gelfand transform, and $d \hat{f}(x) / d x$ exists for almost all $x$.

Proposition 1.2. If $E \subseteq(0,1]$ is closed, and $f \in L^{1}(0,1)$ has $\hat{f}(e)=0$ for all $e \in E$, then there exist $f_{n} \in L^{1}(0,1)$ such that $\left\|f * f_{n}-f\right\|_{1} \rightarrow 0$ and $\hat{f}_{n}$ is zero in a neighborhood of $E$.

Comment. This proposition asserts that every closed set is a set of spectral synthesis for $L^{1}(0,1)$, in spite of the fact that $L^{1}(0,1)$ consists of almost everywhere differentiable functions; many examples of failure of spectral synthesis use differentiation (e.g. $\left\{\frac{1}{2}\right\}$ is not a set of synthesis for $\left.C^{1}(0,1)\right)$; thus, Proposition 1.2 is slightly surprising.

The proof of Proposition 1.2 requires the following lemma.

LEMMA 1.3. If $\hat{f}(x)=0$ for $x \in E$, then $\int_{E}|f(t)| d t=0$.

Proof. If $x_{0} \in E$, and $x_{n} \in E$, we have $\lim x_{n}=x_{0}$, then

$$
0=\lim \left(\hat{f}\left(x_{n}\right)-\hat{f}\left(x_{0}\right)\right) /\left(x_{n}-x_{0}\right)
$$

so $d \hat{f} / d t=0$ almost everywhere on $E$, since $\left\{\hat{f}: f \in L^{1}(0,1)\right\}$ is the set of functions $g$ on $(0,1]$ such that (a) $d g / d t$ exists almost everywhere, (b) $\int_{0}^{1}|d g / d t|<\infty$, and (c) $g(x)=\int_{0}^{x}(d g / d t) d t$. Thus $0=\int_{E}|d \hat{f}| d t\left|d t=\int_{E}\right| f(t) \mid d t$. Q.E.D.

ProOF of Proposition 1.2. By using the lemma, we may conclude that $E=\bigcap_{n=1}^{\infty} E_{n}$ where each $E_{n}$ is a finite union of mutually disjoint intervals which are closed in $(0,1]$, that each $E_{n}$ contains a neighborhood of 0 ,

$$
\lim _{n \rightarrow \infty} \int_{E_{n}}|f| d t=0
$$


and that all the endpoints of all the intervals which make up each $E_{n}$ belong to $E$.

Let $\varepsilon>0$ be fixed, and choose $n$ so large that

$$
\int_{E_{n}}|f|<\varepsilon / 4 \text {. }
$$

Now $E_{n}=\left(0, b_{1}\right] \cup\left[a_{2}, b_{2}\right] \cup \cdots \cup\left[a_{k}, b_{k}\right]$ where $0<b_{1}<a_{2}<b_{2}<\cdots<$ $a_{k}<b_{k} \leqq 1$.

Choose numbers $b_{j}^{\prime}, b_{j}^{\prime \prime}$ and $a_{j}^{\prime}, a_{j}^{\prime \prime}$ such that $a_{j}^{\prime}<a_{j}^{\prime \prime}<a_{j}, b_{j}<b_{j}^{\prime \prime}<b_{j}^{\prime}$, $\int_{a_{j}^{\prime}}^{a_{j}}|f|<\varepsilon / 8 k, \int_{b_{j}^{\prime}}^{b^{\prime}}|f|<\varepsilon / 8 k, j=1,2, \cdots, k$.

Let $\chi(a, b)$ denote the characteristic function of $[a, b]$, and set

$$
g=-\sum_{j=2}^{k}\left(a_{j}^{\prime \prime}-a_{j}^{\prime}\right)^{-1} \chi\left(a_{j}^{\prime}, a_{j}^{\prime \prime}\right)+-\sum_{j=1}^{k}\left(b_{j}^{\prime \prime}-b_{j}^{\prime}\right)^{-1} \chi\left(b_{j}^{\prime}, b_{j}^{\prime \prime}\right)
$$

(the second sum runs only to $k-1$ if $b_{k}=1$ ). It is easy to see that $\hat{g}=0$ on $\left(0, b_{1}^{\prime}\right] \cup\left[a_{2}^{\prime \prime}, b_{2}^{\prime}\right] \cup \cdots \cup\left[a_{k}^{\prime \prime}, b_{k}^{\prime}\right]$ (last term is $\left[a_{k}^{\prime \prime}, 1\right]$ if $\left.b_{k}=1\right)$ and $\hat{g}=1$ on $\left[b_{1}^{\prime \prime}, a_{2}^{\prime}\right] \cup \cdots \cup\left[b_{k-1}^{\prime \prime}, a_{k}\right] \cup\left[b_{k}^{\prime \prime}, 1\right]$ (the last term does not appear if $b_{k}=1$ ). Thus $\hat{g}$ is zero on a neighborhood of $E_{n}$.

Furthermore, a straightforward calculation shows $\|g * f-f\|<\varepsilon$. [Indeed,

$$
\|g * f-f\|=\int_{0}^{b_{1}{ }^{\prime \prime}}|g * f-f|+\int_{a_{2}^{\prime}}^{b_{2}{ }^{\prime \prime}}|g * f-f|+\cdots .
$$

We compute a sample term: between $a_{2}^{\prime}$ and $b_{2}^{\prime \prime}$,

$$
\begin{aligned}
\int_{a_{2}^{\prime}}^{b_{2}{ }^{\prime \prime}}|g * f-f| & =\int_{a_{2^{\prime}}}^{b_{2}^{\prime \prime}}\left|\frac{1}{a_{2}^{\prime \prime}-a_{2}^{\prime}} \chi\left(a_{2}^{\prime}, a_{2}^{\prime \prime}\right) * f-f\right| \\
& \leqq \int_{a_{2}^{\prime}}^{b_{2}^{\prime \prime}}\left|\frac{1}{a_{2}^{\prime \prime}-a_{2}^{\prime}} \chi\left(a_{2}^{\prime}, a_{2}^{\prime \prime}\right) * f\right|+\int_{a_{2^{\prime}}}^{b_{2}^{\prime \prime}}|f| \\
& \leqq \int_{a_{2^{\prime}}}^{b_{2}^{\prime \prime}}|f|+\int_{a_{2^{\prime}}}^{b_{2}^{\prime \prime}}|f| \leqq 2 \int_{a_{2^{\prime}}}^{a_{2}}|f|+2 \int_{a_{2}}^{b_{2}}|f|+2 \int_{b_{2}}^{b_{2}^{\prime \prime}}|f|
\end{aligned}
$$

so

$$
\left.\|g * f-f\| \leqq 2 \sum_{j=1}^{k}\left(\frac{\varepsilon}{8 k}+\frac{\varepsilon}{8 k}\right)+2 \int_{E_{n}}|f|<\frac{2 \varepsilon}{4}+\frac{2 \varepsilon}{4}=\varepsilon .\right] \quad \text { Q.E.D. }
$$

2. Proof of result (1). Here the algebra $\mathscr{A}$ is the tensor product $\mathscr{A}=L^{1}(0,1) \hat{\otimes} L^{1}(Z)$; that is, $\mathscr{A}$ is the set of integrable functions on $(0,1) \times Z$. The product on $\mathscr{A}$ is the tensor product, so the maximal ideal space of $\mathscr{A}$ is $(0,1] \times T$, where $T$ is the circle group. It is obvious that $\mathscr{A}$ is a commutative convolution measure algebra and that $\mathscr{A}^{\wedge}$, the set of Gelfand transforms of $\mathscr{A}$, is a regular algebra on $(0,1] \times T$. 
Choice of $E_{1}$ and $E_{2}$. Let $x_{1}=\frac{1}{2}$, and let $\left\{x_{j}: j=2,3, \cdots\right\}$ be an increasing sequence in $(0,1)$ with $\lim x_{j}=\frac{1}{2}$. Let $\left\{y_{j} \in T\right\}_{j=2}^{\infty}$ be an infinite set of distinct elements with one accumulation point $y_{1}$ and such that $\left\{y_{j}\right\}_{1}^{\infty}$ is an interpolation set for $L^{1}(Z)$. Define

$$
\begin{aligned}
& E_{1}=\left\{\left(x_{j}, y_{j}\right): j=1,2, \cdots\right\}, \quad \text { and } \\
& E_{2}=\left\{\left(x_{1}, y_{1}\right)\right\} \cup\left\{\left(x_{j+1}, y_{j}\right): j=2,3, \cdots\right\} .
\end{aligned}
$$

That $E_{1}$ and $E_{2}$ are interpolation sets follows from $\S 1$.

$E_{1} \cup E_{2}$ is not an interpolation set: Functional analysis arguments show that it will be enough to prove that for each integer $N=1,2, \cdots$ there exists $f \in C\left(E_{1} \cup E_{2}\right)$ such that if $g \in \mathscr{A}$ and $\hat{g}=f$ on $E_{1} \cup E_{2}$ then $\|g\| \geqq$ $(N / 2)\|f\|_{\infty}[10]$, where $\|f\|_{\infty}=\sup _{x}|f(x)|$.

Fix $N \geqq 1$, and let $f$ be a continuous function on $E_{1} \cup E_{2}$ of supremum norm one, and such that

$$
f\left(x_{j}, y_{j}\right)=0 ; f\left(x_{j+1}, y_{j}\right)=1 \quad \text { for } j=2,4,6, \cdots, 2 N .
$$

Suppose $g \in \mathscr{A}$ interpolates $f$.

Choose points $x_{j}<\bar{x}_{j}<x_{j+1}$ (for $j=2,3, \cdots, 2 N+2$ ) and let $\bar{x}_{1}=0$, $\bar{x}_{2 N+3}=1$. Then there exist $g_{1}, \cdots, g_{2 N+2} \in \mathscr{A}$ with

$$
g=\sum_{1}^{2 N+2} g_{j} \text { and }\|g\|_{\mathscr{A}}=\sum\left\|g_{j}\right\|_{\mathscr{A}}
$$

and

$$
\text { support } g_{j} \subseteq\left[\bar{x}_{j}, \bar{x}_{j+1}\right] \times Z, \quad j=1, \cdots, 2 N+2 .
$$

From (2) and (4) we conclude

$$
\begin{aligned}
x \geqq \bar{x}_{j+1} \Rightarrow \hat{g}_{j}(x, y)=\hat{g}_{j}\left(x_{j+1}, y\right), \\
x \leqq \bar{x}_{j} \Rightarrow \hat{g}_{j}(x, y)=0 .
\end{aligned}
$$

Therefore if $j$ is even and less than $2 N$, we have so

$$
\begin{aligned}
& 0=\hat{g}\left(x_{j}, y_{j}\right)=\hat{g}_{1}\left(x_{1}, y_{j}\right)+\cdots+\hat{g}_{j-1}\left(x_{1}, y_{j}\right)+\hat{g}_{j}\left(x_{j}, y_{j}\right), \\
& 1=\hat{g}\left(x_{j+1}, y_{j}\right)=\hat{g}_{1}\left(x_{1}, y_{j}\right)+\cdots+\hat{g}_{j}\left(x_{1}, y_{j}\right)+\hat{g}_{j+1}\left(x_{j+1}, y_{j}\right),
\end{aligned}
$$

$$
1=\hat{g}_{j}\left(x_{1}, y_{j}\right)+\hat{g}_{j+1}\left(x_{j+1}, y_{j}\right)-\hat{g}_{j}\left(x_{j}, y_{j}\right) .
$$

The last formula implies

or

$$
1 \leqq 2\left\|g_{j}\right\|_{\mathscr{A}}+\left\|g_{j+1}\right\|_{\mathscr{A}}
$$

$$
\frac{1}{2} \leqq\left\|g_{j}\right\|_{\mathscr{A}}+\left\|g_{j+1}\right\|_{\mathscr{A}} \quad \text { for } j=2,4,6, \cdots, 2 N \text {. }
$$

From (6) and (3) we conclude that $\|g\|_{\mathscr{A}} \geqq N / 2$. 
Remark. For $C^{1}([0,1]) \times C([0,1])=\mathscr{A}$, it is easy to see that $\{(1 / n, 1 / n): n=1,2, \cdots\} \cup\{(0,0)\}$ and $\{(0,1 / n): n=1,2, \cdots\} \cup\{(0,0)\}$ are interpolation sets whose union is not an interpolation set.

3. Proof of result (2). The result follows at once from:

TheOREM 3.1. Let $\mathscr{A}=L^{1}\left(R^{2}\right) \hat{\otimes} L^{1}(0,1)$. Then (i) $\mathscr{A}$ is a regular Banach algebra with maximal ideal space $X=\boldsymbol{R}^{2} \times(0,1]$.

(ii) if $x \in X, f \in \mathscr{A}$, and $\hat{f}(x)=0$, then there exist $f_{n} \in \mathscr{A}$ such that $\lim \left\|f_{n} f-f\right\|_{\mathscr{A}}=0$ and $\hat{f}_{n} \equiv 0$ in a neighborhood of $x$.

(iii) There exists a compact arc $E \subseteq X$ such that $E$ is an interpolation set for $\mathscr{A}$ and such that spectral synthesis fails for $E$.

Proof. (i) This follows at once from general considerations (see $\$ 1$ and [14, p. 58]) and Proposition 1.1.

(ii) Let $f=\sum_{1}^{\infty} g_{j} \otimes h_{j} \in \mathscr{A}$ and $x=\left(x_{1}, x_{2}\right)$ have $0=\hat{f}(x)=\sum_{1}^{\infty} g_{j}\left(x_{1}\right) \hat{h}_{j}\left(x_{2}\right)$, and $2\|f\|_{\mathscr{A}} \geqq \sum\left\|g_{j}\right\|\left\|h_{j}\right\|$. Fix $\varepsilon>0$, and let $N$ be so large that

$$
\sum_{j>N}\left\|g_{j}\right\|\left\|h_{j}\right\|<\varepsilon / 8
$$

From 1.2 and the corresponding fact for $L^{\mathbf{1}}\left(\boldsymbol{R}^{2}\right)[10,2.6]$, we know that there exists for each $\delta>0, \tilde{g}_{1}, \cdots, \tilde{g}_{N} \in L^{1}\left(R^{2}\right)$ and $\tilde{h}_{1}, \cdots, \tilde{h}_{N} \in L^{1}(0,1)$ such that for $j=1, \cdots, N,\left\|g_{j}-\tilde{g}_{j}\right\|<\delta,\left\|h_{j}-\tilde{h}_{j}\right\|<\delta, \tilde{g}_{j}^{\wedge}=\hat{g}_{j}\left(x_{1}\right)$ in a neighborhood of $x_{1}$, and $\tilde{h}_{j}^{\hat{\beta}}=\hat{h}_{j}\left(x_{2}\right)$ in a neighborhood of $x_{2}$. Then for $\delta>0$ sufficiently small,

$$
\left\|\sum_{1}^{N} g_{j} \otimes h_{j}-\sum_{1}^{N} \tilde{g}_{j} \otimes \tilde{h}_{j}\right\| \leqq \varepsilon / 8 .
$$

Now choose $g \in L^{1}\left(R^{2}\right)$ and $h \in L^{1}\left(R^{2}\right)$ such that $\hat{g}=1$ in a neighborhood of $x_{1}, \hat{h}=1$ in a neighborhood of $x_{2}$, and $\|g\| \leqq 2,\|h\| \leqq 2$. Then

$$
k=\sum_{1}^{N} \tilde{g}_{j} \otimes \tilde{h}_{j}-\left(\sum_{j>N} \hat{g}_{j}\left(x_{1}\right) \tilde{h}_{j}\left(x_{2}\right)\right) g \otimes h
$$

is zero in a neighborhood of $\left(x_{1}, x_{2}\right)$ and

$$
\|f-k\| \leqq\left\|\sum_{1}^{\infty} g_{j} \otimes h_{j}-k\right\| \leqq \varepsilon / 8+\varepsilon / 8+4(\varepsilon / 8)=6 \varepsilon / 8<\varepsilon .
$$

Before proving (iii) we prove

LEMmA 3.2. Let $f \in \mathscr{A}$. Then there exists a measurable set $Z \subseteq(0,1]$ of zero measure such that $\partial \hat{f}(x, y, z) / \partial z$ exists for all $(x, y, z) \in \boldsymbol{R}^{2} \times((0,1] \backslash Z)$, and

$$
\int_{0}^{1}\left|\sup _{(x, y) \in R^{2}} \frac{\partial \hat{f}}{\partial z}(x, y, z)\right| d z \leqq\|f\| .
$$


Proof. To avoid confusion, denote $\partial \hat{f} / \partial z$ by $D \hat{f}$, and let $\varepsilon>0$. Then write $f=\sum_{1}^{\infty} g_{j} \otimes h_{j}$ where $\sum\left\|g_{j}\right\|\left\|h_{j}\right\| \leqq\|f\|+\varepsilon$. Of course

$$
\hat{f}(x, y, z)=\sum_{1}^{\infty} \hat{g}_{j}(x, y) \hat{h}_{j}(z)
$$

so

$$
D \hat{f}=\sum_{1}^{\infty} \hat{g}_{j}(x, y) \frac{d \hat{h}_{j}}{d z}(z)
$$

whenever this last expression makes sense. Now for each $j=1,2, \cdots$ there exists a measurable set $Z_{j} \subset(0,1]$ of measure zero such that $d \hat{h}_{j} / d z$ exists for all $z \notin Z_{j}$. Set $Z_{0}=\bigcup_{j=1}^{\infty} Z_{j}$. For $z \notin Z_{0}$,

$$
\left|\sum \sup _{(x, y)} \hat{g}_{j}(x, y) \frac{d \hat{h}_{j}}{d z}(z)\right| \leqq \sum\left\|g_{j}\right\|\left|\frac{d \hat{h}_{j}}{d z}(z)\right| .
$$

Note that $\int_{0}^{1} \sum\left\|g_{j}\right\|\left|d \hat{h}_{j} / d z\right| d z=\sum\left\|g_{j}\right\|\left\|h_{j}\right\| \leqq\|f\|+\varepsilon$, so that except for a measurable set $Z \subseteq(0,1]$ of measure zero, the sum (3.1) converges for all $(x, y) \in \boldsymbol{R}^{2}$ and $z \in(0,1], z \notin Z$. This completes the proof of 3.2.

Proof of (iii). Let $\varphi:\left[\frac{1}{2}, 1\right] \rightarrow R^{2}$ be a continuous function whose image is a Helson (interpolation) set for $A\left(R^{2}\right)=L^{1}\left(R^{2}\right)^{\wedge}$. That such functions exist has been proved by Kahane [6] (see also McGehee [9]).

Let $E=\left\{(x, y, z) \in \boldsymbol{R}^{2} \times(0,1]:(x, y)=q(z), \frac{1}{2} \leqq z \leqq 1\right\}$. Then $E$ is an interpolation set for $\mathscr{A}$.

To show that $E$ is not a set of spectral synthesis, we show that $E$ supports an element $\mu$ of $\mathscr{A}^{*}$ which is not a "measure", that is, the $\operatorname{map} f \mapsto \mu(f)$ is not bounded in the spectral radius norm sup $|\hat{f}|=\|\hat{f}\|_{\infty}$. We then show (Lemma 3.4) that if a subset $F$ of the maximal ideal space of a (regular commutative) Banach algebra $\mathscr{B}$ is both an interpolation set and a set of spectral synthesis, then every element, $v \in \mathscr{B}^{*}$ which is supported on $F$ is a measure.

Lemma 3.3. E supports $\mu \in \mathscr{A}^{*}$ such that $f \rightarrow \mu(f)$ is not bounded in the norm $\|\hat{f}\|_{\infty}$.

Proof. For $\hat{f} \in \mathscr{A}^{\wedge}$, let $D \hat{f}(x, y, z)$ denote the partial derivative of $\hat{f}$ with respect to the third variable. If $f=\sum g_{j} \otimes h_{j}$, then

$$
D \hat{f}(x, y, z)=\sum \hat{g}_{j}(x, y) h_{j}(z) .
$$

By 3.2, Dरf $(x, y, z)$ exists whenever $z \notin Z$, a fixed (depending on $f$ ) set of measure zero. For $k \in L^{\infty}(0,1)$, and $f \in \mathscr{A}$, define $S_{k}(f)$ by

$$
S_{k}(f)=\int_{1 / 2}^{1} D \hat{f}(\varphi(z), z) k(z) d z .
$$


For $f=\sum g_{j} \otimes h_{j}$, and $\|f\|+\varepsilon \geqq \sum\left\|g_{j}\right\|\left\|h_{j}\right\|$, we see

$$
\left|S_{k}(f)\right| \leqq \sum\left\|g_{j}\right\| \int\left|h_{j} k\right| d z \leqq\|k\|_{\infty}(\|f\|+\varepsilon) .
$$

Thus $S_{k} \in \mathscr{A}^{*}$ and $\left\|S_{k}\right\| \leqq\|k\|_{\infty}$. If $\hat{f} \equiv 0$ in a neighborhood of $E$, then $D f \equiv 0$ in a neighborhood of $E$, so $S_{k}$ is concentrated on $E$.

Note that (3.2) implies that

$$
S_{k}(1 \otimes h)=\int_{1 / 2}^{1} h(z) k(z) d z \quad\left(h \in L^{1}(0,1)\right)
$$

It is easy to see that there exist $k \in L^{\infty}(0,1)$ such that $f \mapsto S_{k}(f)$ is not $\|\hat{f}\|_{\infty}$-bounded. [Indeed, let $k_{0}(z)=\cos (1 /(1-z))$, and

$$
\begin{aligned}
h_{n}(z) & =(1-z)^{-2} \cos (1 /(1-z)), & & \text { if } \pi(1-z) \geqq(2 n+1)^{-1}, \\
& =0, & & \text { otherwise. }
\end{aligned}
$$

A straightforward integration shows $\sup \left\{\left|\hat{h_{n}}(z)\right|: n \geqq 1, z \in(0,1)\right\}$ is bounded, and a second integration shows $\left|\int h_{n} k_{0} d z\right|$ is not bounded. Now let $f_{n}=1 \otimes h_{n}$ and apply (3.3).] Q.E.D.

The next lemma seems to be known, though we are unable to give a reference.

Lemma 3.4. Let $\mathscr{B}$ be a regular commutative Banach algebra with identity and maximal ideal space $X$. Suppose $F \subseteq X$ is both an interpolation set and a set of spectral synthesis, and that $v \in \mathscr{B} *$ is supported by $F$. Then for some constant $C<\infty$,

for all $f \in \mathscr{B}$.

$$
|v(f)| \leqq C \sup \{|\hat{f}(x)|: x \in F\}
$$

Proof. For $f \in \mathscr{B}$, and $g \in \mathscr{B}$, with $\hat{g}$ zero everywhere on $F$, there exist $g_{n} \in \mathscr{B}, \hat{g}_{n} \equiv 0$ in a neighborhood of $F$, and $\left\|g-g_{n}\right\| \rightarrow 0$. Then

$$
v(f+g)=\lim _{n} v\left(f+g_{n}\right)=\lim \left(v(f)+v\left(g_{n}\right)\right)=v(f)+\lim 0
$$

since $v$ is supported by $F$. Thus

$$
|v(f)| \leqq\|v\| \inf \{\|f+g\|: \hat{g}(F)=\{0\}\} .
$$

Since $F$ is an interpolation set and $\mathscr{B}$ is regular, there is a constant $C^{\prime}$ such that, for all $f \in \mathscr{B}$,

$$
\inf \{\|f+g\|: \hat{g}(F)=\{0\}\} \leqq C^{\prime} \sup \{|f(x)|: x \in F\} .
$$

Putting (3.5) and (3.4) together, and setting $C=\|v\| C^{\prime}$, we obtain the desired inequality. Q.E.D.

This completes the proof of Theorem 3.1(ii). 
Remarks. (a) By using $L^{1}\left(Z^{x}\right)$ instead of $L^{1}\left(R^{2}\right)$, we can construct a "Kronecker" set for $\mathscr{A}^{\prime}=L^{1}\left(Z^{\infty}\right) \hat{\otimes} L^{1}(0,1)$ which is not a set of spectral synthesis. The algebra $\mathscr{A}^{\prime}$ will also be regular and symmetric on its maximal ideal space $T^{\infty} \times[0,1]$. The set to be constructed will be

$$
E=\left\{\left(\left(e^{i x}, e^{i x^{2}}, \cdots\right), x\right):{ }_{3}^{1} \leqq x \leqq \frac{2}{3}\right\} .
$$

(b) It is easy to see that if $\mathscr{A}^{\prime}=L^{1}\left(R^{2}\right) \hat{\bigcup} L^{1}(R)$ (where the second factor is given the multiplication $x \cdot y=\max (x, y)$, then the conclusion of Theorem 1 holds for $\mathscr{A}^{\prime}$, and that $\mathscr{A}^{\prime}$ is strongly homogeneous (in the terminology of [9]) with respect to the group $\Phi$ of homeomorphisms of $\boldsymbol{R}^{3}$ which are of the form $\varphi(x, y, z)=(f(x, y), g(z))$ where $f: \boldsymbol{R}^{2} \rightarrow \boldsymbol{R}$, and $g: \boldsymbol{R} \rightarrow \boldsymbol{R}$ are affine.

(c) The method of the proof of Theorem 3.1 shows that

$$
E=\left\{(x, x): \frac{1}{2} \leqq x \leqq 3\right\}
$$

is not a set of spectral synthesis for $B=L^{1}(0,1) \hat{\otimes} L^{1}(0,1)$. Thus, very simple sets fail, in general, to be sets of synthesis for the tensor product $B$. Compare [15].

4. Proof of result (3). We will (eventually) use the same computation used to establish result (1). The tensor method is somewhat obscured by the technical details. We first set forth the method, and then establish the necessary properties.

Suppose we have distinct characters $\gamma_{1}, \gamma_{2}, \cdots \in \hat{G}$ such that every bounded function on $\left\{\gamma_{j}\right\}_{1}^{\infty}$ may be obtained as the restriction of the Fourier (-Stieltjes) transform of a discrete measure on $G$ to $\left\{\gamma_{j}\right\}_{1}^{\text {x }}$. Suppose also that $p_{1}, p_{2}, \cdots$ are distinct projections of $M(G)$ onto $L$-subalgebras of $M(G)$ such that if $v$ is a discrete measure, then $p_{j} v=v$ for all $j$, and such that if $\mu \in M(G)$, then

$$
\begin{gathered}
\mu=\mu-p_{1} \mu+\sum_{j=1}^{\infty}\left(p_{j}-p_{j+1}\right) \mu ; \\
\|\mu\|=\left\|\mu-p_{1} \mu\right\|+\sum_{j=1}^{\infty}\left\|\left(p_{j}-p_{j+1}\right) \mu\right\|,
\end{gathered}
$$

$\mu \geqq 0$ implies $p_{j}(\mu) \geqq 0$; and such that each $p_{j}$ is multiplicative. (We shall construct the $p_{j}$ by using Raikov systems [3].)

Let $\chi_{j}(\mu)=\int \gamma_{j} d\left(p_{j} \mu\right)$ and $\rho_{j}(\mu)=\int \gamma_{j} d\left(p_{j+1} \mu\right)$. Then $\psi_{j}$ and $\rho_{j}$ are continuous multiplicative linear functionals on $M(G)$, and they are pairwise distinct. It is now easy to show that if $E_{1}$ is the closure of $\left\{\rho_{j}\right\}$ and $E_{2}$ the closure of $\left\{\chi_{j}\right\}$ in the maximal ideal space of $M(G)$, then $E_{1}$ and $E_{2}$ are interpolation sets for $M(G)$, and their union is not, since any $\mu \in M(G)$ 
with

$$
\hat{\mu}\left(\chi_{2 j}\right)=1, \quad \hat{\mu}\left(\rho_{2 j}\right)=0 \quad \text { for } j=1, \cdots, N
$$

must have norm $N$. Just apply (4.2) and the definition of $\chi_{j}$ and $\rho_{j}$ to obtain

$$
1=\int \gamma_{j} d\left(p_{2 j}-p_{2 j+1}\right) \mu=\left(\left(p_{2 j}-p_{2 j+1}\right) \mu\right)^{\wedge}\left(\gamma_{j}\right)
$$

so $\left\|\left(p_{2 j}-p_{2 j+1}\right) \mu\right\| \geqq 1$. Now (4.1) implies $\|\mu\| \geqq N$.

We now show how to obtain $\gamma_{j}$ and $p_{j}$.

For the $\gamma_{j}$ 's this has been done by Varopoulos [17]. We indicate his method. It will be enough to choose the $\gamma_{j}$ 's such that every function on $\left\{\gamma_{j}\right\}_{1}^{\infty}$ which takes only the values \pm 1 may be approximated to within onethird by a character in $G$. When $\hat{G}$ contains an element of infinite order $\gamma$ which generates a discrete subgroup, then an easy computation shows $(j !) \gamma=\gamma_{j}$ will do. If $\hat{G}$ contains an independent set $\left\{\gamma_{j}\right\}$ which generates a discrete subgroup of $\hat{G}$, then $\left\{\gamma_{j}\right\}_{1}^{\infty}$ will do. If $G$ contains a perfect Kronecker set, $K$, then the $\gamma_{j}$ may be constructed inductively: divide $K$ into six perfect nonempty subsets and choose $\gamma_{1}$ such that $\gamma_{1}$ is approximately constant on each subset and $\gamma_{1}(K)$ (nearly) contains all sixth roots of unity. Divide each of the six sets obtained for $\gamma_{1}$ into six perfect subsets, and choose $\gamma_{2}$ such that $\gamma_{2}$ is (approximately) constant on each of the thirtysix subsets and such that $\gamma_{2}$ maps each of the first six sets (approximately) onto the sixth roots of unity, etc.

We now construct the $p_{j}$ 's. We have two cases: (i) $G$ contains a perfect totally disconnected metrizable compact Kronecker set $F$; (ii) $G$ contains a perfect totally disconnected metrizable compact set $F$ of type $K_{p}, p \geqq 2$.

For both cases we fix $x_{0} \in F$, and choose closed-open subsets $F_{j}$ of $F$ such that $F_{1} \supseteq F_{2} \supseteq \cdots ; F_{j} \backslash F_{j+1}$ is perfect, $\bigcap_{1}^{\infty} F_{j}=\left\{x_{0}\right\}$.

Also, for both cases we let $R_{j}, j=1,2, \cdots$, be the Raikov (regular) system [3] generated by $F_{j}$, and $p_{j}$ be the projection of $M(G)$ onto the $L$ subalgebra of measures concentrated on sets in $R_{j}$. Then [3] $p_{1}, p_{2}, \cdots$ have all of the required properties, except that they might not be distinct.

In case (i), the argument is straightforward: if $\mu$ is a continuous nonzero measure on $F_{j} \backslash F_{j+1}$, then $\mu$ has zero mass on every translate of sums of $F_{j+1}$ with itself so $p_{j+1} \mu=0$ and $p_{j} \mu \neq 0$; see [10], [12] for details.

In case (ii), the situation is more complicated, owing to the fact that the section arguments used in [10], [12] do not always apply (directly, at any rate) because of the finite order of the group.

We first prove:

Lemma 4.1. Let $A \cup B$ be a compact, totally disconnected metrizable subset of an LCAG $G$ of type $K_{q}$ with $A \cap B=\varnothing$, and $A, B$ both closed in $G$. 
Then the closed subgroups $H, K$ generated by $A, B$ respectively meet only at the identity.

Proof. We may suppose that $G=D_{q}$ and that the group generated by $A \cup B$ is dense in $G$.

Suppose there is a net $y^{\alpha}(\alpha \in I)$ such that $y^{\alpha}=\sum_{j=1}^{j} n_{j}^{\alpha} x_{j}^{\alpha}$ where $x_{j}^{\alpha} \in A$, and $y=\lim y^{\alpha}$ exists and is in $K$. We must show $y=0$.

Since $G=D_{q}$, we may assume $1 \leqq n_{j}^{\alpha}<q$ for all $j$, and all $\alpha$. For $m=$ $1,2, \cdots, q$, let $I_{m}=\left\{\alpha: \sum_{j} n_{j}^{\alpha} \equiv m(q)\right\}$. If some $I_{m}$, for $1 \leqq m<q$, is cofinal in $I$, let $\gamma \in \hat{D}_{q}$ be equal to $\exp \left(2 \pi i q^{-1}\right)$ on $A$ and 1 on $B$. Then $\lim _{\alpha \in I_{m}}\left(\gamma, y^{\alpha}\right)=(\gamma, y)=\exp \left(2 \pi i m q^{-1}\right) \neq 1$ so $y \notin K$. Thus, only $I_{q}$ is cofinal in $I$.

Since $A \cup B$ is totally disconnected, we can repeat the preceding argument for any of the many proper closed-open subsets $A^{\prime} \subseteq A$ : if $I_{m}^{\prime}=$ $\left\{\alpha:\left\{\sum n_{j}^{\alpha}: x_{j}^{\alpha} \in A^{\prime}\right\} \equiv m(q)\right\}$, then only $I_{q}^{\prime}$ is cofinal. Thus, if $\gamma$ is any character on $H$, then $\left(y^{\alpha}, \gamma\right) \rightarrow 1$, so $y=0$. Q.E.D.

Corollary. If $x \in G$, then $H \cap(K+x)$ is one point.

Proof. If $u, v \in H \cap(K+x)$, then $u=h=k+x$, so $x \in H+K=H \oplus K$. Also if $v=h^{\prime}=k^{\prime}+x \in H \cap(K+x)$, then $x=h-k=h^{\prime}-k^{\prime}$, so $h=h^{\prime}, k=$ $k^{\prime}$ and $u=v$. Q.E.D.

We now prove that the projections $p_{j}$ are distinct. Let $p_{j}^{\prime}(j=1,2, \cdots)$ denote the projection of $M(G)$ onto the algebra supported by the Raikov system generated by the closure of the group $K_{j}$ generated by $F_{j}$. Then $p_{j}^{\prime} p_{j}=p_{j} p_{j}^{\prime}=p_{j}$, since the Raikov system giving rise to $p_{j}^{\prime}$ contains that giving rise to $p_{j}$. Thus, to show $p_{j} \neq p_{j+1}$, it will be sufficient to find a measure $\mu$ with $p_{j} \mu=\mu$ and $p_{j+1}^{\prime} \mu=0$.

Let $\mu$ be any continuous measure concentrated on $F_{j} \backslash F_{j+1}$. Then the preceding corollary (with $A=F_{j} \backslash F_{j+1}$ and $B=F_{j+1}$ ) implies that $|\mu|\left(K_{j+1}+x\right)=0$ for all $x \in G$ and $K_{j+1} \supseteq F_{j+1}$. By the definition of a Raikov system, we see that $p_{j} \mu=\mu$ and $p_{j+1}^{\prime} \mu=0$. Q.E.D.

\section{REFERENCES}

1. J. Benedetto, (LF)-spaces and distributions on compact groups and spectral synthesis on $R / 2 \pi Z$, Math. Ann. 194 (1971), 52-67.

2. S. W. Drury, Sur les ensembles parfaits et les séries trigonométriques, C. R. Acad. Sci. Paris Sér. A-B 271 (1970), A94-A95. MR 42 \#6529.

3. I. M. Gel'fand, D. A. Raǐkov and G. E. Šilov, Commutative normed rings, Fizmatgiz, Moscow, 1960; English transl., Chelsea, New York, 1964. MR 23 \#A1242; MR 34 \#4940.

4. C. S. Herz, Drury's Lemma and Helson sets, Studia Math. 42 (1972), 205-219.

5. E. Hewitt and H. S. Zuckerman, Structure theory for a class of convolution algebras, Pacific J. Math. 7 (1957), 913-941. MR 19, 435. 
6. J.-P. Kahane, Séries de Fourier absolument convergentes, Springer, Berlin, 1970. MR 43 \#801.

7. T. W. Körner, Sets of synthesis for general algebras, Proc. Cambridge Philos. Soc. 67 (1970), 559-568. MR 41 \#830.

8. - Two results on very strongly homogeneous algebras, Proc. Cambridge Philos. Soc. 68 (1970), 363-376. MR 41 \#8994.

9. O. C. McGehee, Helson sets in $T^{n}$, Conference in Harmonic Analysis, Lecture Notes in Math., vol. 266, Springer-Verlag, Berlin, 1972.

10. W. Rudin, Fourier analysis on groups, Interscience Tracts in Pure and Appl. Math., no. 12, Interscience, New York, 1962. MR 27 \#2808.

11. S. Saeki, Spectral synthesis for the Kronecker sets, J. Math. Soc. Japan 21 (1969), 549-563. MR 40 \#7733.

12. A. B. Simon, The ideal space and Silov boundary of a subalgebra of measures on a group, J. Math. Anal. Appl. 6 (1963), 266-276. MR 26 \#6804.

13. J. L. Taylor, The structure of convolution measure algebras, Trans. Amer. Math. Soc. 119 (1965), 150-166. MR 32 \#2932.

14. N. Th. Varopoulos, Groups of continuous functions in harmonic analysis, Acta. Math. 125 (1970), 109-152.

15. —_ Tensor algebras and harmonic analysis, Acta. Math. 119 (1967), 51-112. MR 39 \#1911.

16. - Sur les ensembles parfaits et les séries trigonométriques, C. R. Acad. Sci. Paris 260 (1965), 3831-3834. MR 32 \#322.

17. - The functions that operate on $B_{0}(\Gamma)$ of a discrete group $\Gamma$, Bull. Soc. Math. France 93 (1965), 301-321. MR 34 \#565.

18. T. W. Körner, A pseudofunction on a Helson set, Asterisque (to appear).

Department of Mathematics, Northwestern University, Evanston, Illinois 60201 